YAROSLAV SHRAMKO

HEINRICH WANSING

\section{Editorial Introduction. Truth Values: Part I}

The present special issue of Studia Logica is devoted to the topic of truth values, which is at the very heart of many important problems of modern symbolic logic and analytic philosophy. Truth values have been put to quite different uses in philosophy and logic, being characterized, for example, as:

- primitive abstract objects denoted by sentences in natural and formal languages,

- abstract entities hypostatized as the equivalence classes of sentences,

- values indicating the degree of truth of sentences,

- entities that can be used to explain the vagueness of concepts,

- values that are preserved in valid inferences,

- entities that convey information concerning a given proposition.

Depending on their particular use, truth values can be treated as unanalyzed, as defined, as unstructured, or as structured entities. These entities prove to be an effective instrument for a logical and semantical analysis of language, as well as for the representation and explication of various sophisticated problems arising in many-valued logics, partial logics, fuzzy logics, paraconsistent logics, etc., which are heavily oriented towards possible applications in epistemology, AI, computational linguistic and other related fields. The papers included in this special issue put into focus some of these key problems and thoroughly discuss manifold roles truth values can play in logical investigations.

The notion of a truth value has been explicitly introduced by Gottlob Frege - for the first time in [10], and most notably in his seminal paper [11]. Frege considered exactly two classical truth values, the True (das Wahre) and the False (das Falsche), which played in his theory the role of references (Bedeutungen) for sentences. In this way truth values can be understood as specific objects, whereas sentences are interpreted as a certain kind of proper names destined to denote (refer to) these objects. However, in one significant respect the idea of truth values contravenes traditional approaches to truth by bringing to the forefront the problem of its categorial classification. In most of the established conceptions, truth is usually treated as a property. It is customary to talk about a "truth predicate" and its attribution to sentences, propositions, beliefs or the like. Such an understanding corresponds 
also to a routine linguistic practice, when one operates with the adjective 'true' and asserts, e.g., 'that 5 is a prime number is true'. By contrast with this apparently quite natural attitude, the suggestion to interpret truth as an object may seem rather confusing, to say the least. Nevertheless this suggestion is equipped with a profound and strong motivation demonstrating that it is far from being just an oddity and has to be taken seriously.

Nuel Belnap in his paper "Truth values, neither-true-nor-false and supervaluations" calls the conception of truth values as denotations of sentences "a happy idea" and argues that we ought not to give up the Fregean truth values in favor of a Tarskian truth predicate. It is emphasized that truth values as semantic values for sentences allow for many useful formal parallels to other entities serving as denotations of singular terms. This is elucidated in some detail for substitution. Moreover, Belnap considers a number of auxiliary parameters to which truth can be relativized. In this part of the paper, applications of the supervaluation technique are considered and various kinds of supervaluation are compared with each other, including Tarski's treatment of variables in first-order logic. The paper concludes with the advice not to confuse "supervalues" and real truth values.

As it has been observed repeatedly in the literature, the stress Frege laid on the notion of a truth value was, to a great extent, pragmatically motivated. Besides an intended gain for his system of "Basic Laws" reflected in enhanced technical clarity, simplicity, and unity, Frege also sought to substantiate in this way his view on logic as a theoretical discipline with truth as its main goal and primary subject-matter. According to such a view, the proper task of logic consists, ultimately, in investigating "the laws of being true". Now, if one admits that truth is represented by a specific abstract object (the corresponding truth value), then logic in the first place has to explore the features of this object and its interrelations to other entities of various other kinds. A prominent adherent of this conception was also Jan Eukasiewicz, who considered logic to be the science of objects of a special kind, namely the science of logical values, where truth can be seen as the positive, and falsehood is the negative logical value (see [14, p. 90]).

If truth values are accepted and taken seriously as a special kind of objects, the obvious question as to the nature of these entities arises. The above characterization of truth values as objects is far too general and requires further specification. One way of such concretization is to qualify truth values as abstract objects. In particular, truth values can be introduced by applying so-called abstraction principles, which are used for supplying abstract 
objects with criteria of identity. For truth values such a criterion can be given by stating that for any two sentences $p$ and $q$, the truth value of $p$ is identical with the truth value of $q$ if and only if $p$ is equivalent with $q[4,7]$. In this way truth values obtain a certain ontological substantiation.

One can also find in the literature another strategy of explicating the very nature of truth values by providing them with a kind of epistemic justification. According to this strategy, a truth value of a sentence is generally conceived as representing certain information about this sentence supplied from some information source(s). The information can be just of the sort "this sentence is true", or "this sentence is false", but it is also possible to take into account information of a more complex character. One can consider, e.g., an epistemic situation when information about the sentence is simultaneously received from several different sources.

The paper by Melvin Fitting, "How true it is = who says it is true", in a way contributes to this epistemic interpretation. It is proposed to identify the truth value of a formula with the set of agents who accept this formula (as true). Subsequently, Fitting takes an arbitrary (finite) Boolean algebra as an underlying truth value space. The resulting logical framework turns out to be a many-valued modal logic, which is provided with both a generalized Kripke semantics and a deductive machinery in the form of a complete tableau calculus. The paper contains a case study of common knowledge that uses the Muddy Children Puzzle as an example. Moreover, bisimulations for the languages under consideration are considered and an effective algebraic criterion whether two frames are bisimilar is presented.

The foundations for many-valued logics have been laid down by Łukasiewicz, who proposed to take seriously other logical values different from truth and falsehood. By generalizing this idea and also adopting the above understanding of the subject-matter of logic, one naturally arrives at the wellknown representation of particular logical systems as a certain kind of valuation systems.

Consider a propositional language $\mathcal{L}$ built upon a set of atomic sentences and a set of propositional connectives $\mathcal{C}$. Then a valuation system $\mathbf{V}$ for the language $\mathcal{L}$ is a triple $\langle\mathcal{V}, \mathcal{D}, \mathcal{F}\rangle$, where $\mathcal{V}$ is a non-empty set with at least two elements, $\mathcal{D}$ is a non-empty proper subset of $\mathcal{V}$, and $\mathcal{F}=\left\{f_{c_{1}}, \ldots f_{c_{m}}\right\}$ is a set of functions such that $f_{i}$ is an $n$-place function on $\mathcal{V}$ if $c_{i}$ is an $n$-place connective. Intuitively, $\mathcal{V}$ is the set of truth values, $\mathcal{D}$ is the set of designated truth values, and $\mathcal{F}$ is the set of truth-value functions interpreting 
the elements of $\mathcal{C}$. If the set of truth values of a valuation system $\mathbf{V}$ has $n$ elements, $\mathbf{V}$ is said to be $n$-valued. Valuation systems are usually referred to as (logical) matrices. The set $\mathcal{D}$ of designated values is of central importance for the notion of a valuation system. This set represents a generalization of the classical truth value $T$ (the True) determining thus many other logical notions, the notions of a tautology and the entailment relation among them.

The elements of $\mathcal{V}$ are sometimes referred to not as "truth values" but as "truth degrees". The term "truth degrees", used by many authors working in the field of many-valued logic, suggests that truth comes by degrees, and these degrees may be seen as truth values in an extended sense. The idea of truth as a graded notion has been applied to model vague predicates, and the vagueness of concepts was one of the major motivations for the development of fuzzy logic. In the 1960ies, Lotfi Zadeh [22] introduced the notion of a fuzzy set. Whereas the range of the characteristic function of a classical set is the two-element set $\{0,1\}$ (which may be seen as the set of classical truth values), a fuzzy set has a membership function ranging over the real interval $[0,1]$. Vague predicates may then be regarded as denoting fuzzy sets. The membership function of the fuzzy set denoted by some vague predicate assigns thus values (seen as truth degrees) from the interval $[0,1]$.

Petr Hájek in his paper "On vagueness, truth values and fuzzy logics" aims at showing that the role of the fuzzy logic in the study of vagueness should not be underestimated. The focus of his consideration is the book by Steward Shapiro [16], where several important topics related to the concept of vagueness are discussed, the Sorites Paradox, supervaluations, tolerance and open-texture among them. Hájek demonstrates how Shapiro's apparatus can be fruitfully combined with fuzzy logic, possibly generalized by interval-valued interpretations (generalizing Kleene's extension of classical logic).

One might, perhaps, think that the mere existence of many-valued logics shows that there exist infinitely, in fact, uncountably many truth values. However, this is not at all self-evident (recall the more cautious terminology of "truth degrees"). Moreover, in the 1970ies Roman Suszko declared that many-valued logic is "a magnificent conceptual deceit" [19, p. 377]. Suszko claimed that "there are but two logical values, true and false", a statement now called Suszko's Thesis. For Suszko, the set of truth values assumed in a logical matrix for a many-valued logic is a set of "admissible referents" (called "algebraic values") of formulas but not a set of logical values. Whereas the algebraic values are elements of an algebraic structure and referents of 
formulas, the logical value true is used to define valid consequence: If every premise is true, then so is (at least one of) the conclusion(s). The other logical value, false, is preserved in the opposite direction: If the (every) conclusion is false, then so is at least one of the premises. The logical values are thus represented by a bi-partition of the set of algebraic values into a set of designated values (truth) and its complement (falsity), see also [20, 21].

Josep Maria Font ("Taking degrees of truth seriously") takes Suszko's thesis as the starting point in his discussion of the role of truth degrees in many-valued logics from the perspective of Abstract Algebraic Logic. Font argues that one of the ways to explicate Suszko's distinction between "algebraic values" and "logical values" is to interpret them, respectively, as the truth values of a metatheory and the truth values of a theory. This interpretation can serve as a natural framework for a consideration of the logics that preserve degrees of truth. The paper presents a number of such logics and highlights the need for further philosophical motivation in the selection of the truth values that should be taken to determine the degrees of truth.

Suszko's thesis is substantiated by a rigorous proof (the Suszko Reduction) showing that every consequence relation of a certain kind and therefore also every many-valued propositional logic of such kind is characterized by a bivalent semantics. The dichotomy between designated values and values which are not designated and its use in the definition of entailment plays a crucial role in the Suszko Reduction. Nevertheless, while it seems quite natural to construe the set of designated values as a generalization of the classical truth value $T$, it would not always be adequate to interpret the set of non-designated values as a generalization of the classical truth value $F$. The point is that in a many-valued logic, unlike in classical logic, "not true" does not always means "false".

In the literature on many-valued logic it is sometimes proposed to consider a set of antidesignated values which does not obligatorily constitute the complement of the set of designated values. The set of antidesignated values can be regarded as representing a generalized concept of falsity. This distinction leaves room for values that are neither designated nor antidesignated and even for values that are both designated and antidesignated.

Such an approach is congenial to a certain strategy of generalizing classical truth values initiated in some works by J. Michael Dunn (see, e.g. [8]). According to this strategy a sentence can be rationally considered to be not just true or just false, but also neither true nor false as well as both 
true and false. The corresponding semantic construction ought to admit undetermined and overdetermined valuations often called "truth value gaps" and "truth value gluts" respectively. Nuel Belnap [2, 3] takes Dunn's idea a step further by explicitly regarding the gaps and the gluts as new truth values. Using a highly heuristic (epistemic) interpretation of a truth value as information that "has been told to a computer" he arrived at a "useful four-valued logic" of "how a computer should think" with the following four generalized truth values: (1) N - none ("told neither falsity nor truth"); (2) $\mathbf{F}$ - "plain" falsehood ("told only falsity"); (3) $\mathbf{T}$ - "plain" truth ("told only truth"); (4) B - both falsehood and truth ("told both falsity and truth"). These four truth values form a certain valuation system with $\mathbf{T}$ and $\mathbf{B}$ as designated values (and possibly $\mathbf{F}$ and $\mathbf{B}$ as antidesignated values).

Generalized truth values have a strong intuitive background, especially as a tool for the rational explication of incomplete and inconsistent epistemic situations. But the conception of generalized truth values has its purely logical import as well. Belnap's generalized truth values can be represented as the power-set of the set of Frege's two truth values. If one continues the construction and applies the idea of such generalization to Belnap's four truth values, then one obtains the set of 16 generalized truth values representing all the possible combinations of $\mathbf{N}, \mathbf{F}, \mathbf{T}$ and $\mathbf{B}$. This new set of values has been investigated in $[17,18]$, where among other things it has been shown how it can serve as a natural semantic foundation for the logic of a simple computer network.

As soon as we admit that truth values come with valuation systems, it is quite natural to assume that the elements of such a system are somehow interrelated. And indeed, already the valuation system for classical logic constitutes a well-known algebraic structure, namely the two-element Boolean algebra with $\cap$ and $\cup$ as meet and join operators. In its turn, this Boolean algebra forms a lattice with a partial order defined by $a \leq_{t} b$ iff $a \cap b=a$. It is easy to see that the elements of this lattice are ordered as follows: $F \leq_{t} T$. This ordering is sometimes called the truth order (as indicated by the corresponding subscript), for intuitively it expresses an increase in truth: $F$ is "less true" than $T$. The relation $\leq_{t}$ is also called a logical order, because it can be used to determine key logical notions: logical connectives and an entailment relation. Namely, if the elements of the given valuation system $\mathbf{V}$ form a lattice, then the operations of meet and join with respect to $\leq_{t}$ are usually seen as the functions for conjunction and disjunction, whereas negation can be represented by the inversion of this order. Moreover, one can consider an entailment relation for $\mathbf{V}$ as expressing agreement with the truth order. Another prominent partially ordered 
valuation system is Belnap's valuation system mentioned above. The set of truth values $\{\mathbf{N}, \mathbf{T}, \mathbf{F}, \mathbf{B}\}$ constitutes a specific algebraic structure - the bilattice FOUR $R_{2}$ (see, e.g., [9]). This bilattice is equipped with two partial orderings; in addition to a truth order, there is an information order $\left(\leq_{i}\right)$ which is said to order the values under consideration according to the information they give concerning a formula to which they are assigned.

It turns out that the 16 values arising from a generalization of Dunn's and Belnap's logic give rise to an algebraic structure with three distinct partial orders: an information order $\leq_{i}$ (viz. set-inclusion), a truth order $\leq_{t}$ and a falsity order $\leq_{f}$. Whereas the truth order is defined in terms of the presence and absence of the classical value $T$, the falsity order is defined in terms of the presence and absence of $F$. The resulting algebraic structure is known as the trilattice SIXTEEN $N_{3}$. It turns out that while in the bilattice FOU $R_{2}$ the logical order is not merely a truth order, but rather a truth-and-falsity order (an increase in truth means here a simultaneous decrease in falsity), the trilattice $S I X T E E N_{3}$ makes it possible to discriminate between a truth order and a (non-)falsity order. This means that in $S I X T E E N_{3}$, in addition to the information order, we have actually two distinct logical orders: one for truth, $\leq_{t}$, and one for falsity, $\leq_{f}$. Both of these logical orderings determine their own logic, in a truth vocabulary (where entailment is defined with respect to $\leq_{t}$ ), and in a falsity vocabulary (where entailment is defined with respect to $\leq_{f}$ ). For both languages one obtains first-degree entailment as the logic of SIXTEEN $N_{3}$, see [17]. In [18] this observation has been generalized to trilattices of any degree.

Sergei Odintsov in "On axiomatizing Shramko-Wansing's logic" presents a solution to the problem of constructing an axiomatic system in the full language with the truth and falsity connectives for truth-entailment in $S I X T E E N_{3}$. This solution is based on a semantic representation of $S I X T E E N_{3}$ as a so-called (four-component) "twist-structure" over the two-element Boolean algebra. To obtain an axiomatization, the language of $[17,18]$ is enriched with an implication connective which is interpreted as the residuum of truth-conjunction with respect to the truth order of SIXTEEN $N_{3}$. The presence of implication allows Odintsov to apply a result on axiomatizing the intersection of two intermediate logics presented as axiom systems with modus ponens as the only rule of inference.

Gottlob Frege's notion of a truth value has become part of the standard philosophical and logical terminology. This notion is an indispensable tool of realistic, model-theoretic approaches to semantics, and therefore truth 
values also play an essential role in applications of model-theoretic semantics in areas such as, for example, knowledge representation and theorem proving based on semantic tableaux. There are, however, certain concerns whether the Fregean truth values are anything more than just a convenient technical tool. Do we have any reason to believe that there really is any such thing as a truth value? In view of this questions it is instructive to observe that Frege provides truth values (as proper referents of sentences) not merely with a pragmatical motivation but also with a strong theoretical justification. The idea of such justification, that can be found in [12, p. 49], employs the principle of substitutivity of co-referential terms (known also as the principle of compositionality or extensionality), according to which the reference of a complex singular term must remain unchanged when any of its sub-terms is replaced by an expression having the same reference. And as Frege asks: "What else but the truth value could be found, that belongs quite generally to every sentence if the reference of its components is relevant, and remains unchanged by substitutions of the kind in question?"

This idea has been implemented into a formal argument by Church [5], Gödel [13], Davidson [6] and others with the intention to provide a strict proof for the claim that all true sentences designate (denote, refer to) one and the same thing, as well as all false sentences do. These things are precisely the truth values: the True and the False. This line of reasoning is now widely known under the name "slingshot argument", a term coined by Jon Barwise and John Perry in [1, p. 395]. The slingshot argument has been analyzed in detail by many authors, see especially the most comprehensive study by Stephen Neale [15] and references therein, and has caused much controversy notably on the part of "fact-theorists", i.e. adherents of facts, situations, propositions, states of affairs and other fact-like entities conceived as alternative candidates for denotations of sentences. Not to speak of its purely logical import, this argument has many far reaching philosophical consequences, in particular for the correspondence conception of truth, factontologies, theories of modalities, etc.

"The slingshot argument and sentential identity" by Yaroslav Shramko and Heinrich Wansing explores the analysis of the slingshot argument by means of a non-Fregean logic as developed by Roman Suszko. It is demonstrated that the language of non-Fregean logic can serve as an illuminating instrument for reconstructing and fruitfully analyzing the slingshot argument. The paper unfolds several versions of the slingshot argument in non-Fregean logic enriched either with operators of definite or indefinite descriptions or with the lambda-abstractor. Moreover, 
it presents a new version of the slingshot argument, which can be circumvented neither by an appeal to a Russellian theory of definite descriptions nor by resorting to an analogous "Russellian" theory of $\lambda$-terms.

This special issue of Studia Logica is based on submissions received after a call for papers and on some invited lectures presented at the International Workshop on Truth Values, held from May 29 - June 1, 2008, at the Institute of Philosophy, Dresden University of Technology, Germany, see 〈http://www.truthvalues2008.com/〉. The speakers at the workshop were: Arnon Avron (Tel Aviv), Nuel Belnap (Pittsburgh), Jean-Yves Béziau (Neuchâtel), Walter Carnielli (São Paulo), Michael Dunn (Bloomington), Melvin Fitting (New York), Siegfried Gottwald (Leipzig), Petr Hájek (Prague), Norihiro Kamide (Tokyo), Beata Konikowska (Warsaw), Saul Kripke (New York), Grzegorz Malinowski (Łódź), Sergei Odintsov (Novosibirsk), Yaroslav Shramko (Krivoi Rog), and Heinrich Wansing (Dresden).

Altogether we received 21 submissions, each of which underwent a strict anonymous refereeing, and we would like to thank the referees for their excellent work and assistance. From the invited workshop presentations, the paper by Norihiro Kamide and the joint paper by Beata Konikowska and Arnon Avron will appear in regular issues of Studia Logica, other papers in Part II. The workshop in Dresden was generously supported by The German Research Council (DFG), grant WA 936/6-1, by the German Society for Analytic Philosophy (GAP) and by the Gesellschaft von Freunden und Förderern der Technischen Universität Dresden. We gratefully acknowledge this sponsorship. Moreover, we would like to thank Jacek Malinowski for supporting the idea of a Studia Logica special issue devoted to truth values.

\section{References}

[1] Barwise, J., and J. Perry, 'Semantic innocence and uncompromising situations', Midwest Studies in the Philosophy of Language VI (1981), 387-403.

[2] Belnap, N.D., 'How a computer should think', in G. Ryle (ed.), Contemporary Aspects of Philosophy, Oriel Press Ltd., Stocksfield, 1977, pp. 30-55.

[3] Belnap, N.D., 'A useful four-valued logic', in J.M. Dunn and G. Epstein (eds.), Modern Uses of Multiple-Valued Logic, Reidel, Dordrecht, 1977, pp. 8-37.

[4] Carnap, R., Meaning and Necessity. A Study in Semantics and Modal Logic, University of Chicago Press, Chicago, 1947.

[5] Church, A., 'Review of Rudolf Carnap, Introduction to Semantics', The Philosophical Review 52 (1943), 298-304.

[6] Davidson, D., 'Truth and meaning', Synthese 17 (1967), 304-323.

[7] Dummetr, M., 'Truth', in Truth and Other Enigmas, Harvard University Press, Cambridge, Massachusetts, 1978, pp. 1-24. 
[8] Dunn, J.M., 'Intuitive semantics for first-degree entailment and 'coupled trees', Philosophical Studies 29 (1976), 149-168.

[9] Fitting, M., 'Bilattices are nice things', in T. Bolander, V. Hendricks, and S.A. Pedersen (eds.), Self-Reference, CSLI-Publications, Stanford, 2006, pp. 53-77.

[10] Frege, G., 'Function und Begriff. Vortrag, gehalten in der Sitzung vom 9. Januar 1891 der Jenaischen Gesellschaft für Medicin und Naturwissenschaft', Jena: H. Pohle, Jena, 1891, 31 pp. (Reprinted in [12].)

[11] Frege, G., 'Über Sinn und Bedeutung', Zeitschrift für Philosophie und philosophische Kritik 100 (1892), 25-50. (Reprinted in [12].)

[12] Frege, G., Funktion, Begriff, Bedeutung. Fünf logische Studien, G. Patzig (ed.), Vandenhoeck \& Ruprecht, Göttingen, 1986.

[13] GöDel, K., 'Russell's mathematical logic', in P.A. Schilpp (ed.), The Philosophy of Bertrand Russell, Northwestern University Press, Evanston and Chicago Ill., 1944, 125-153.

[14] Łukasiewicz, J., Selected Works, L. Borkowski (ed.), North-Holland \& PWN, Amsterdam \& Warsaw, 1970.

[15] Neale, S., Facing Facts, Oxford University Press, Oxford, 2001.

[16] Shapiro, S., Vagueness in Context, Oxford University Press, Oxford, 2006.

[17] Shramko, Y., and H. Wansing, 'Some useful 16-valued logics: how a computer network should think', Journal of Philosophical Logic 34 (2005), 121-153.

[18] Shramko, Y., and H. Wansing, 'Hypercontradictions, generalized truth values, and logics of truth and falsehood', Journal of Logic, Language and Information 15 (2006), 403-424.

[19] Suszko, R., 'The Fregean axiom and Polish mathematical logic in the 1920's', Studia Logica 36 (1977), 373-380.

[20] Wansing, H., and Y. Shramko, 'Suszko's Thesis, inferential many-valuedness, and the notion of a logical system', Studia Logica 88 (2008), 405-429, 89 (2008), 147.

[21] Wansing, H., and Y. Shramko, 'Harmonious many-valued propositional logics and the logic of computer networks', in C. Dégremont, L. Keiff and H. Rückert (eds.), Dialogues, Logics and Other Strange Things. Essays in Honour of Shahid Rahman, College Publications, London, 2008, pp. 491-516.

[22] ZaDeH, L., 'Fuzzy sets', Information and Control 8 (1965), 338-353.

YAROSLAV SHRAMKO

Department of Philosophy

State Pedagogical University

50086 Krivoi Rog, Ukraine

shramko@rocketmail.com
HEINRICH WANSING

Institute of Philosophy

Dresden University of Technology

01062 Dresden, Germany

Heinrich. Wansing@tu-dresden.de 